\title{
Designing an information resource to explain diagnostic lumbar puncture and promote best practice
}

\author{
Alison Thomson ${ }^{1}$, Angharad Davis ${ }^{1}$, Adam Paterson ${ }^{2}$, Gavin Giovannoni ${ }^{1}$, Klaus Schmierer ${ }^{1}$ \\ ${ }^{1}$ Queen Mary, University of London, ${ }^{2}$ The Agency of Design
}

INTRODUCTION:

The lumbar puncture (LP) procedure is an important test in neurological practice. It is also being used to assess outcome indices in clinical trials.

Appropriate information is key to both preparing patients for this invasive procedure and facilitating use of atraumatic LP needle systems by clinicians in order to minimize adverse effects such as local pain and post-lumber puncture headache.

\section{FEEDBACK FROM A PATIENT:}

"Finally, the letter for my LP admission. It contains all the things I must and mustn't do prior to my appointment, but tells me nothing about after the procedure. As a patient I want to know:

- Can I go home on my own?

- How long does it take?

- How long do I have to stay there after it has been done?

- Can I return to work?

...I still don't know what to expect afterwards, so am left to Google the procedure, which is never a good thing."

\section{OUR RESPONSE:}

A cross-disciplinary team (consisting of designers, clinicians and people with multiple sclerosis, pwMS) designed and developed a website to explain the LP procedure at The Royal London Hospital (Barts Health NHS Trust, UK).

\section{The design and development process:}

The information presented includes what to expect during the procedure, how to prepare for it, how to deal with adverse effects, the purpose of examining cerebrospinal fluid and information on the atraumatic needle.

Illustrations and animations were incorporated to present the information in an engaging format.

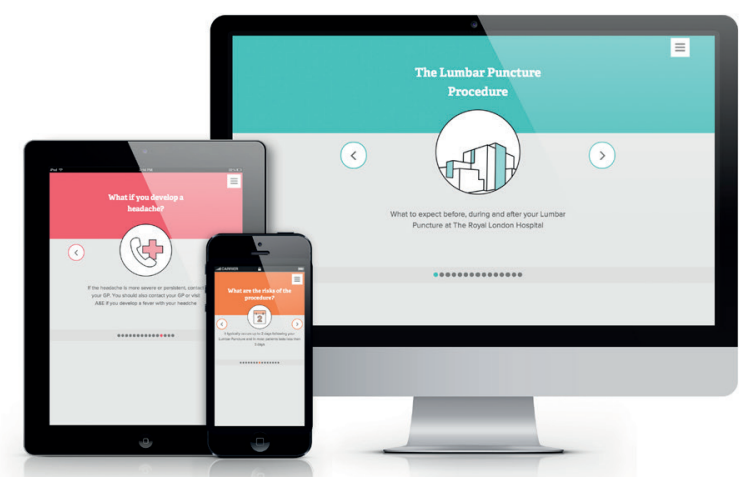

The information resource was designed as a fully responsive website to work across multiple technology platforms.

User testing was carried out with the Barts MS Advisory Group to review both the visual design (colour contrast, legibility) and functionality (navigation and usability) of the resource for people who commonly suffer from visual and cognitive limitations.

\section{Use \& Dissemination:}

\section{PROXIMUS TRIAL RECRUITMENT}

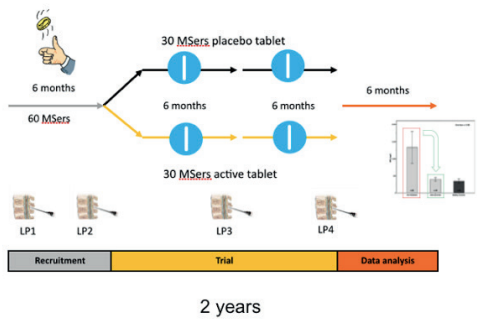

The website is being used as part of the informed consent process for clinical trials involving pwMS at our institution. We are also currently pilqting the use of the tool in our day unit and A\&E departments in patients awajting $P$.
THE PATIENT CHAMPION

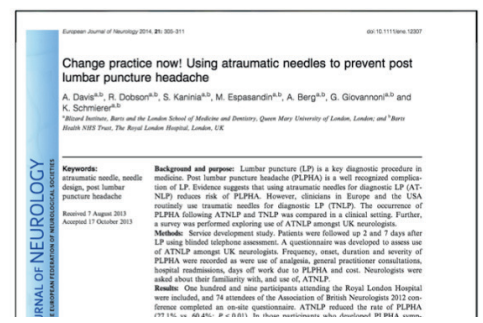

It also demonstrates an innovative way of spreading best practice in hospitals and encourages the widespread adoption of atraumatic needles for LP in both clinical and research settings.
HELP US CHANGE PRACTICE

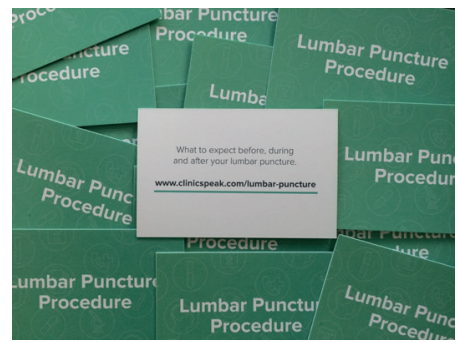

The resource provides an appropriate way of communicating sensitive, yet important information to patients prior to the LP procedure.

Distrobute business cards...

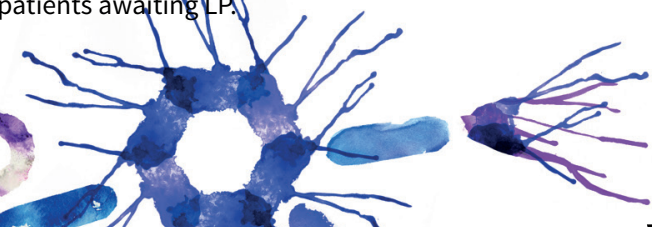

